\title{
A New Transmission Tariff Allocation Model based on Bilevel Programming
}

\author{
Érica Telles, Delberis A. Lima, Javier Contreras, Fellow, IEEE, Natalia Alguacil, Senior Member,
} IEEE

\begin{abstract}
This paper proposes a flow-based approach for the transmission tariff allocation problem based on the notions of long run marginal cost (LRMC) and nodal exchange factors (NEFs). A worst-case power flow, expressed in terms of NEFs, is formulated to characterize the system state. Additionally, the NEFs optimal values are determined considering a specific criterion to compute tariffs. The resulting problem is formulated as a bilevel program. The upper level is a worst-case power flow that maximizes line flows taking into account the transmission tariffs computed in the lower level. The criterion considered in the lower level aims to minimize the variation range of transmission tariffs, which is particularly desirable to provide incentives for renewable sources installed far away from load centers. The resulting bilevel problem may have multiple solutions. Here, the uniqueness in tariffs is preserved by solving an alternative problem once both the generation dispatch and the variation range of transmission tariffs are known. Numerical results are provided for a 6-bus system and the IEEE 118-bus system.
\end{abstract}

Index Terms - Transmission tariff allocation, bilevel optimization, long run marginal cost, renewable generation.

\section{NOMENCLATURE}

\section{A. Indices}

$g$

$g$

d

B. Sets

$\Omega_{b}$

$\Omega_{g}$

$\Omega_{d}$

$\Omega_{g}^{b}$
Index of buses

Index of generators

Index of demands

Index of transmission lines

Set of buses

Set of generators

Set of demands

Set of generators located at bus $b$

\section{$d_{\ell}$}

This work was suppored in part by the Ministry of Science of Spain, and by the Ministry of Economy and Competitiveness of Spain, Project ENE201563879-R (MINECO/FEDER, UE), and the Junta de Comunidades de Castilla - La Mancha under Project POII-2014-012-P. The author E. Telles received a scholarship from CAPES under process BEX 8871/14-0. The author Delberis

A. Lima acknowledges the partial financial support from FAPERJ (Project $\mathrm{n}^{\circ}$ E-26/102.999/2012) and CNPq (Project n ${ }^{\circ} 478837 / 2013-5$ ).

E. Telles and D.A. Lima are with the Department of Electrical Engineering at the Pontifical Catholic University of Rio de Janeiro, Rio de Janeiro, Brazil (e-mail: erica.tcarlos@gmail.com; delberis@ele.puc-rio.br).

J. Contreras and N. Alguacil are with the E.T.S. de Ingenieros Industriales, University of Castilla - La Mancha, Ciudad Real 13071, Spain (e-mail: Javier.Contreras@uclm.es; Natalia.Alguacil@uclm.es).
$\Omega_{d}^{b}$

$\Omega_{\ell}$

C. Constants

$\beta_{\ell b}$

$C_{\ell}$

$C_{\ell}^{T}$

$\mathrm{Cap}_{\ell}$

$\gamma_{g d e}$

$P_{d}$

$P_{g}^{M A X}, P_{g}^{M I N}$

$\Delta^{G E N}, \Delta^{D E M}$

$T_{g}, T_{d}$

$Z^{M A X}, W^{M I N}$

$N^{G E N}, N^{D E M}$

$N^{L I N}$

D. Variables

$f_{\ell}$

$f_{\ell}^{g, d}$

$p_{g}$

$\alpha_{g d}^{G E N}, \alpha_{d g}^{D E M}$

Set of demands located at bus $b$

Set of transmission lines

Sensitivity of line $\ell$ due to a power injection/extraction at bus $b$ for a given slack bus

Per unit cost of line $\ell$

Total cost of line $\ell$

Transmission capacity of line $\ell$

Distribution factor (DF) of line $\ell$ due to a power injection at a bus where generator $g$ is located and extraction at a bus where demand $d$ is located

Peak value of demand $d$

Upper and lower limits of power production by generator $g$, respectively

Postage stamp tariff for generators and for demands, respectively

Total tariff for generator $g$ and demand $d$, respectively

Upper and lower tariff bounds, respectively

Number of generators and demands, respectively

Number of transmission lines

\section{DC power flow through line $\ell$}

Power flow component through line $\ell$ due to a power injection at a bus where generator $g$ is located and extraction at a bus where demand $d$ is located

Weighting factor for line $\ell$

Power output of generator $g$

Generation and demand nodal exchange factors between the generator $g$ and demand $d$, respectively

$\pi_{g}, \pi_{d} \quad$ Locational tariffs for generator $g$ and demand $d$, respectively

$y_{W P F} \quad$ Value of the objective function of the worstcase power flow.

Transmission tariff allocation objective function

Auxiliary variables to represent the tariffs upper and lower bounds, respectively

Average of all locational tariffs 


\section{INTRODUCTION}

$\mathrm{T}$ RANSMISSION investments are intended to guarantee reliability and robustness in power systems. In order to recover the cost of such investments, it is necessary to establish a methodology capable of allocating cost quotas to each transmission user, basically generation and distribution utilities. This problem is hereinafter referred to as the Transmission Tariff Allocation (TTA) problem. Since each country has its own regulations and market structures, there is no universal TTA method. In the literature there are several proposals of methods [1]-[19] intending to represent different objectives and expectations of the regulator, users and investors in each country [20]. Their preferences may vary from the socialization of costs [1] to the provision of strong economic signals [2]. In any case, network particularities and constraints must be characterized.

Most transmission networks are in expansion and a deregulated market for generation exists. As a result, locational tariff signals may be viewed as instruments to encourage generation plants to be installed in beneficial areas. The overall idea is to allocate higher/lower tariffs to nodes that contribute more/less to network congestion. To this aim, flowbased allocation methods are widely used.

The evaluation of the transmission usage by flow-based allocation methods may result in multiple solutions due to the diversity of: (i) hypotheses assumed to compute a power flow solution and (ii) power flow decompositions to obtain the transmission tariffs. In the technical literature, the power flow solution and the transmission tariffs are computed separately and sequentially.

In this paper, in order to represent the power flow solution that requires more transmission investments [3], the hypothesis of maximum line congestion is assumed. Hereinafter, this system condition is referred to as worst-case power flow (WPF) setting. Additionally, in order to allocate transmission tariffs based on power flow decomposition, classic solutions are computed by AC power flow expressions [4], by the proportional sharing principle [5], by marginal participation factors [6], by tracing users' flow contribution in a graph representation of the system [7]-[8], or combining wheeling transactions with distribution factors (DF), DC power flow solutions [9]-[11], or using cooperative game theory [12].

When classic methodologies do not succeed in achieving the desired TTA objective, another approach is to define a criterion to be reached, which considers the set of feasible solutions of flow decomposition. Among all the possible decompositions, the best one should properly characterize the expectations of the regulator, users and investors. Power flow tracing has been considered to minimize the deviation from a postage stamp allocation in [13] and to apply a min-max fairness policy in [14]-[15]. The same policy was applied using the feasible sets of distributed slack buses for each transmission user in [16] and [17]. A similar idea was proposed in [18] using an Aumann-Shapley approach. Also, minimum dispersion tariffs were provided in [19] through the feasible set of bilateral power exchanges.
In practice, the TTA criterion may influence the WPF solution. Consequently, solving the WPF and TTA problems in sequence may lead to suboptimal tariffs under the selected TTA criterion.

In systems that are highly congested and/or in expansion, isolated renewable generators, which are limited by the geographic availability of the natural resources, can receive discouraging locational signals in their tariffs by flow-based methods. The high tariffs computed do not stimulate the use of the available, and less costly, renewable sources ([17] and [19]). As an example, when the methods based on long run marginal costs (LRMC) are used, higher tariffs are allocated to isolated renewable generators. In this case, a practical criterion may be to establish tariff bounds and minimize the tariff variation range [19] in order to mitigate the impact of transmission tariffs on isolated generators keeping coherent locational signals.

LRMC represents the additional cost necessary to supply a marginal increment (injection or extraction) of a user in an ideal minimum cost network (see [3] and [17]). In short, the ideal network is the one that can sustain the WPF.

Under a bilevel optimization framework, this paper proposes a general TTA model that allows computing transmission tariffs characterizing their relation with the WPF and the LRMC. The upper level maximizes the lines' congestion (WPF model) taking into account that the transmission tariffs are obtained in the lower level through a LRMC approach. As mentioned before, in this paper, the lower level minimizes the variation range of the tariffs, which can have an influence on the upper level due to the tariff bounds established. The two levels are coupled by both the generation dispatch and the so-called nodal exchange factors (NEFs). The NEF concept was proposed in [17] and [19] to represent fictitious bilateral exchanges between generators and demands as presented in [9].

Depending on the tariff bounds established in the lower level, the solution provided by the bilevel problem may be infeasible, coupled or decoupled. In the case of infeasibility, there is no feasible power flow solution that can meet the tariff bounds in the lower level. If a narrow range of tariffs is established in the lower level, both levels are coupled and the line congestion level is below its maximum attainable value. On the other hand, for a larger range of tariffs, both levels can be decoupled and the TTA problem can be solved independently once the WPF problem is solved. Consequently, the line congestion level achieves its maximum value.

In summary, the main contributions of the paper are:

- To propose a new TTA model considering the combination between the WPF model and the flow decomposition-based method;

- To exploit the flexibility of expressing the power flow solutions through NEFs in order to achieve different regulation criteria for the TTA;

- To propose a framework which is particularly adequate to provide incentives for renewable generation far away from load centers while keeping consistent locational signals; 
- To implement a methodology based on lexicographic optimization to ensure solution uniqueness of the bilevel program.

This paper is organized as follows. Sections II and III present the WPF and TTA models, respectively. Section IV combines both models in the proposed bilevel transmission tariff allocation problem. In this section, the features of the solutions associated to the bilevel model are identified, and a methodology to guarantee solution uniqueness is also presented. In Section V, the proposed framework is analyzed for a 6-bus system and the IEEE 118-bus system. Additionally, a computational performance analysis is presented. In Section VI final conclusions are drawn.

\section{WORST-CASE POWER Flow MOdeL}

As discussed in Section I, the upper level of the proposed TTA bilevel optimization framework is a WPF problem. When flow-based TTA methods are applied, the system state is represented by a power flow solution. This solution may represent a real operating state, the congestion of specific transmission lines, or any other justifiable choice. As mentioned in Section I, in this paper, the tariffs obtained should characterize the system's LRMC. A common practice to ensure the WPF condition is to consider the system's peak demand, dispatching generators proportionally to their capacities. However, the latter condition is not sufficient [3] since the generators' contribution to system congestion is not captured. Thus, the proposed WPF model considers the influence of the generation dispatch decisions in system congestion by solving an optimization problem that maximizes the summation of the linear DC power flows, $f_{\ell}$, in all transmission lines.

Mathematically, the proposed WPF model is formulated as follows:

$\underset{p_{g}, f_{\ell}}{\operatorname{maximize}} \sum_{\ell \in \Omega_{\ell}} f_{\ell}$

subject to:

$f_{\ell}=\sum_{b \in \Omega_{b}} \beta_{\ell b}\left(\sum_{g \in \Omega_{g}^{b}} p_{g}-\sum_{d \in \Omega_{d}^{b}} P_{d}\right) ; \forall \ell \in \Omega_{\ell}$

$\sum_{g \in \Omega_{g}} p_{g}=\sum_{d \in \Omega_{d}} P_{d}$

$P_{g}^{M I N} \leq p_{g} \leq P_{g}^{M A X} ; \quad \forall g \in \Omega_{g}$

$0 \leq f_{\ell} \leq \operatorname{Cap}_{\ell} ; \quad \forall \ell \in \Omega_{\ell}$

The summation of the DC power flows, $f_{\ell}$, through transmission lines is maximized in (1). Constraint (2) defines the power flow using the $\beta_{\ell b}$ elements of the power flow sensitivity matrix [10]. Constraint (3) ensures the system power balance, and (4)-(5) set the generation $\left(P_{g}^{M I N}\right.$ and $\left.P_{g}^{M A X}\right)$ and line capacity $\left(\mathrm{Cap}_{\ell}\right)$ limits, respectively. The presented WPF model is a linear program.

In Section IV the bilevel optimization framework is proposed by merging the WPF model (1)-(5) (upper level) with the TTA model (lower level), which is explained in the next section.

\section{TRANSMISSION TARIFF ALLOCATION MODEL}

As mentioned in Section I, flow-based allocation methods use a solution of the power flow decomposition. In this paper, this solution is obtained through nodal exchange factors (NEFs). An NEF defines a bilateral power exchange between a generator $g$ and a demand $d$, or a pair $(g, d)$. From the generation side, NEF $\alpha_{g d}^{G E N}$ is the percentage of the output of generator $g$ that supplies demand $d$. Analogously, NEF $\alpha_{d g}^{D E M}$ is the percentage of the extraction of demand $d$ supplied by generator $g$ [17]. The NEFs' definition is formulated in (6). From (6), the power flow decomposition is obtained using the distribution factor (DF) concept [9]. The network DF $\gamma_{g d \ell}$ expresses how the power flow of line $\ell$ changes due to a bilateral exchange of $1 \mathrm{MW}$ between the pair $(g, d)$. Thus, the power flow component, $f_{\ell}^{g, d}$, due to a bilateral exchange is defined in (7).

$p_{g} \alpha_{g d}^{G E N}=P_{d} \alpha_{d g}^{D E M} ; \quad \forall g \in \Omega_{g}, \forall d \in \Omega_{d}$

$f_{\ell}^{g, d}=\gamma_{g d \ell} p_{g} \alpha_{g d}^{G E N}=\gamma_{g d \ell} P_{d} \alpha_{d g}^{D E M} ; \quad \forall \ell \in \Omega_{\ell}$

Given the NEF power flow decomposition, a flow-based tariff computation is proposed. The Nodal method [10] and the NEFs are merged in a single formulation. In the Nodal method, which is based on LRMC, all injections/extractions are compensated at the slack bus. Henceforth this assumption will be generalized by the NEFs and a dispersed slack bus is used. From (7), unitary injections $\left(p_{g}\right)$ and extractions $\left(P_{d}\right)$ are considered to obtain the flow-based tariff allocation. The proposed tariff formulations for generators and demands are presented in (8) and (9), respectively. Note that, although a tariff formulation is presented based on the Nodal method, other formulations characterizing NEFs may be used.

$\pi_{g}=\sum_{\ell \in \Omega_{\ell}} \sum_{d \in \Omega_{d}} \frac{C_{\ell}}{2} d_{\ell} \gamma_{g d \ell} \alpha_{g d}^{G E N} ; \quad \forall g \in \Omega_{g}$
$\pi_{d}=\sum_{\ell \in \Omega_{\ell}} \sum_{g \in \Omega_{g}} \frac{C_{\ell}}{2} d_{\ell} \gamma_{g d \ell} \alpha_{d g}^{D E M} ; \quad \forall d \in \Omega_{d}$

From the Nodal method, two terms are adopted: the per unit line costs, $C_{\ell}=C_{\ell}^{T} / C_{a p}$, in $\$ / \mathrm{MW}$, and the line weighting factor, $d_{\ell}=f_{\ell} / \operatorname{Cap}_{\ell}$. Line costs, $C_{\ell}$, are split in two in order to share these costs between generators and demands equally. However, any other sharing rule could be applied. The weighting factor $d_{\ell}$, is used to give more weight to the more congested lines, which contributes to an adequate locational signal. If the line power flow is below a minimum established, $d_{\ell}$ is equal to 0 , which means that this line does not contribute to increase the system marginal transmission costs. Because (8) and (9) are responsible for the locational signals, they are considered locational tariffs.

Since power flows do not generally reach their line capacities, tariffs (8) and (9) do not recover the overall transmission cost. Thus, an additional complementary charge is needed. This charge, also known as postage stamp, is 
formulated for generators and demands in (10) and (11), respectively, based on a pro-rata division of the cost difference.

$\Delta^{G E N}=\frac{\sum_{\ell \in \Omega_{\ell}} \frac{C_{\ell}^{T}}{2}-\sum_{g \in \Omega_{g}} \pi_{g} p_{g}}{\sum_{g \in \Omega_{g}} p_{g}}$

$\Delta^{D E M}=\frac{\sum_{\ell \in \Omega_{\ell}} \frac{C_{\ell}^{T}}{2}-\sum_{d \in \Omega_{d}} \pi_{d} P_{d}}{\sum_{d \in \Omega_{d}} P_{d}}$

Finally, the tariffs applied to generators and demands are as follows:

$T_{g}=\pi_{g}+\Delta^{G E N} ; \quad \forall g \in \Omega_{g}$

$T_{d}=\pi_{d}+\Delta^{D E M} ; \quad \forall d \in \Omega_{d}$

Note that the tariff obtained depends on both the NEFs definition and the TTA criterion adopted. To provide incentives for the installation of renewable generators far away from the demand sites, we propose an optimization problem that minimizes the variation range of locational tariffs within the predefined bounds $Z^{M A X}$ (upper bound) and $W^{M I N}$ (lower bound). The idea is to minimize the highest tariffs and maximize the lowest tariffs. This criterion is intended to provide a more balanced result in order to create the desired incentives. Also, the bounds that can be established by the system regulator ensure the incentives are satisfactory. The TTA model is presented in (14)-(23).

$$
\begin{array}{ll}
\underset{z, w, \alpha_{g d}^{G E N}, \alpha_{d g}^{D E M}}{\operatorname{minimize}}(z-w) & \\
\text { subject to: } & : \theta_{g}^{U} \\
\sum_{\ell \in \Omega_{\ell}} \sum_{d \in \Omega_{d}} \frac{C_{\ell}}{2} d_{\ell} \gamma_{g d \ell} \alpha_{g d}^{G E N} \leq z ; \quad \forall g \in \Omega_{g} & : \varphi_{d}^{U} \\
\sum_{\ell \in \Omega_{\ell}} \sum_{g \in \Omega_{g}} \frac{C_{\ell}}{2} d_{\ell} \gamma_{g d \ell} \alpha_{d g}^{D E M} \leq z ; \quad \forall d \in \Omega_{d} & : \theta_{g}^{L} \\
\sum_{\ell \in \Omega_{\ell}} \sum_{d \in \Omega_{d}} \frac{C_{\ell}}{2} d_{\ell} \gamma_{g d \ell} \alpha_{g d}^{G E N} \geq w ; \quad \forall g \in \Omega_{g} & : \varphi_{d}^{L} \\
\sum_{\ell \in \Omega_{\ell}} \sum_{g \in \Omega_{g}} \frac{C_{\ell}}{2} d_{\ell} \gamma_{g d \ell} \alpha_{d g}^{D E M} \geq w ; \quad \forall d \in \Omega_{d} & : \rho^{L} \\
z \leq Z^{M A X} & : \varepsilon_{g}^{G} \\
\sum_{d} \geq W^{M I N} & \alpha_{g d}^{G E N}=1 ; \quad \forall g \in \Omega_{g} \\
\sum_{g \in \Omega_{g}} \alpha_{d g}^{D E M}=1 ; \quad \forall d \in \Omega_{d} & : \varepsilon_{d}^{D} \\
p_{g} \alpha_{g d}^{G E N}=P_{d} \alpha_{d g}^{D E M} ; \quad \forall g \in \Omega_{g}, \forall d \in \Omega_{d} & : \tau_{g d}
\end{array}
$$

The variation range of the tariffs is minimized in (14), which is formulated as the difference of two auxiliary variables, $z$ and $w$. In (15) and (16), the tariffs' formulation should be lower than $z$, which is minimized in (14). In (17) and (18), tariffs' formulation should be greater than $w$, which is maximized in (14). Hence, the tariffs' range is minimized. The predefined tariff bounds, $Z^{M A X}$ and $W^{M I N}$, are imposed in
(19) and (20). Note that if a range smaller than $Z^{M A X}-W^{M I N}$ is feasible, the lowest possible range can be obtained. As the tariff formulations (8) and (9) are used, there is no need to know to which users the lowest and highest tariffs are allocated. This makes the model independent from the initial solution. Constraints (21)-(23) ensure the power balance of the WPF. The complete TTA model is a linear program. As the postage stamp terms (10) and (11) are functions of the locational tariffs, they are not included in the optimization and they are calculated after the optimization process, as shown in Section IV.C. Note that lower-level dual variables (used in Section IV.A) are given in (15)-(23).

\section{BILEVEL TRANSMISSION TARIFF ALLOCATION}

As proposed in Section I, the WPF and the TTA models are embedded in a bilevel optimization problem. The WPF problem (1)-(5) has an additional set of constraints composed of the minimum variation range tariffs in (14)-(23). The idea is to define a generation dispatch that maximizes the system congestion conditioned by the minimum range of the tariffs. The following sections present the bilevel model, analyze the solution possibilities and provide a procedure to guarantee the solution uniqueness.

\section{A. Bilevel Model}

To obtain a bilevel model from the two models presented in Sections II and III, the line power flow and power balance equations are reformulated according to the NEF concept. Hence, the bilateral exchanges defined by $\alpha_{g d}^{G E N}$ and $\alpha_{d g}^{D E M}$ must be consistent in both levels. Thus, constraints (2) and (3) are replaced by (24) and (25), respectively. In (24) the power flow, $f_{\ell}$, is expressed as the sum of the line flow components, $f_{\ell}^{g, d}$, defined in (7). In (25) the demand is supplied considering the NEF contributions from all generators.

$$
\begin{gathered}
f_{\ell}=\sum_{g \in \Omega_{g}} \sum_{d \in \Omega_{d}} \gamma_{g d \ell} p_{g} \alpha_{g d}^{G E N} ; \quad \forall \ell \in \Omega_{\ell} \\
\sum_{g \in \Omega_{g}} p_{g} \alpha_{g d}^{G E N}=P_{d} ; \quad \forall d \in \Omega_{d}
\end{gathered}
$$

Given the modifications above described, the proposed Bilevel Transmission Tariff Allocation (BTTA) model is as follows:

$$
\begin{aligned}
& \text { Bilevel Transmission Tariff Allocation } \\
& \underset{p_{g}, f_{\ell}}{\operatorname{maximize}} y_{W P F}=\sum_{\ell \in \Omega_{\ell}} f_{\ell} \\
& \text { subject to: } \\
& \text { Constraints (4)-(5) and (24)-(25) } \\
& \text { where } \alpha_{g d}^{G E N} \text { is obtained from: } \\
& \text { minimizize } \\
& z, w, \alpha_{g d}^{G E N}, \alpha_{d g}^{D E M}
\end{aligned}
$$

subject to:

$$
\text { Constraints (15)-(23) }
$$

In order to obtain an equivalent single-level optimization problem for BTTA, results from duality theory in linear 
programming are used. Thus, the linear lower level is replaced by its primal feasibility constraints (15)-(23), the strong duality theorem in (26) and its dual feasibility constraints in (27)-(30).

$$
\begin{aligned}
& \sum_{g \in \Omega_{g}} \varepsilon_{g}^{G}+\sum_{d \in \Omega_{d}} \varepsilon_{d}^{D}-\rho^{U} Z^{M A X}+\rho^{L} W^{M I N}=z-w \\
& \varepsilon_{g}^{G}+\tau_{g d} p_{g}-\theta_{g}^{U} \sum_{\ell \in \Omega_{\ell}} \frac{C_{\ell}}{2} d_{\ell} \gamma_{g d \ell}+ \\
& \quad \theta_{g}^{L} \sum_{\ell \in \Omega_{L}} \frac{C_{\ell}}{2} d_{\ell} \gamma_{g d \ell} \leq 0 ; \forall g \in \Omega_{g}, \forall d \in \Omega_{d} \\
& \varepsilon_{d}^{D}-\tau_{g d} P_{d}-\varphi_{d}^{U} \sum_{\ell \in \Omega_{\ell}} \frac{C_{\ell}}{2} d_{\ell} \gamma_{g d \ell}+ \\
& \varphi_{d}^{L} \sum_{\ell \in \Omega_{\ell}} \frac{C_{\ell}}{2} d_{\ell} \gamma_{g d \ell} \leq 0 ; \forall g \in \Omega_{g}, \forall d \in \Omega_{d} \\
& \sum_{g \in \Omega_{g}} \theta_{g}^{U}+\sum_{d \in \Omega_{d}} \varphi_{d}^{U}-\rho^{U}=1 \\
& \sum_{g \in \Omega_{g}}-\theta_{g}^{L}+\sum_{d \in \Omega_{d}}-\varphi_{d}^{L}+\rho^{L}=-1
\end{aligned}
$$

Regarding the computational complexity of the model, the number of continuous variables is $4+N^{L I N}+4 N^{G E N}+$ $3 N^{D E M}+3 N^{G E N} N^{D E M}$ and the number of constraints is $5+$ $3 N^{L I N}+5 N^{G E N}+4 N^{D E M}+3 N^{G E N} N^{D E M}$. The resulting model is a nonlinear programming problem due to the multiple products of continuous variables.

\section{B. Solution Analysis}

As mentioned in Section III, the BTTA model allows the system regulator to pre-establish bounds for tariffs $\left[W^{M I N}, Z^{M A X}\right]$, which are imposed in (19)-(20). The more restricted the range, the smaller the BTTA feasibility region. Thus, the level of congestion $y_{W P F}$ and parameters $W^{M I N}$ and $Z^{M A X}$ are related, as illustrated in Fig. 1. In order to facilitate the interpretation of this relationship, the value of $W^{M I N}$ is fixed and a two-dimensional graph showing the level of congestion $y_{W P F}$ as a function of $Z^{M A X}$ is presented.

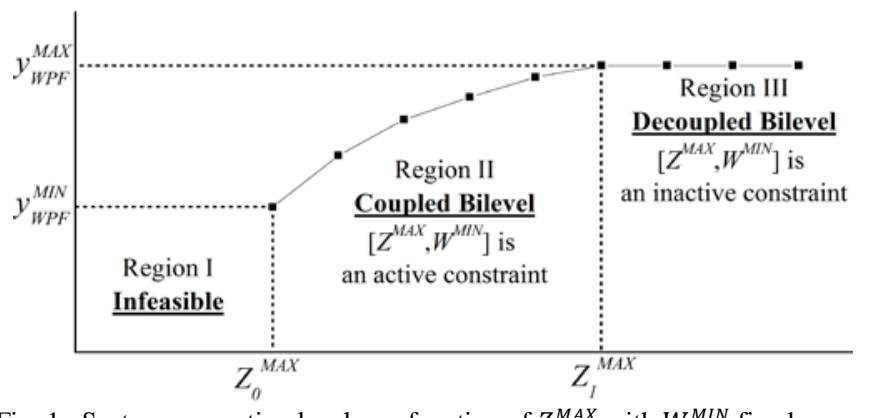

Fig. 1. System congestion level as a function of $Z^{M A X}$ with $W^{M I N}$ fixed.

The solutions of the bilevel problem can be classified as: infeasible (region I), coupled bilevel (region II) and decoupled bilevel (region III). In region I there is no power flow solution that allows the resulting tariffs to be within the bounds $W^{M I N}$ and $Z^{M A X}$. For a given initial value $Z_{0}^{M A X}$ of the upper tariff bound $Z^{M A X}$, the problem becomes feasible and the optimal solution corresponds to the lowest possible value of the maximum congestion level $\left(y_{W P F}^{M I N}\right)$. For larger values of $Z^{M A X}$, up to $Z_{1}^{M A X}$, the bilevel problem is coupled and Fig. 1 can be seen as an efficiency curve (or surface if $W^{M I N}$ varies) of the system congestion level as a function of the tariff bounds. For values of $Z^{M A X}$ larger than $Z_{1}^{M A X}$, the bounds are not restrictive enough to change the upper-level decisions. Thus, the bilevel problem can be decoupled and the highest possible value of the maximum congestion level $\left(y_{W P F}^{M A X}\right)$ is obtained. Since the lower level is not an active constraint, the BTTA can be decoupled and the linear WPF and TTA models presented in Sections II and III can be solved in sequence.

Note that there is no need to know a priori in which region of the curve the tariff bounds are. The BTTA formulation is general enough to capture the three possibilities in Fig. 1.

\section{Solution Uniqueness}

In a realistic transmission system, the number of NEF combinations ( $2 N^{G E N} N^{D E M}$ ) could lead to multiple optimal solutions. To address this issue an extra step in the allocation process is proposed based on lexicographic optimization [21]. In lexicographic optimization, different optimization criteria are ordered by priority. The solution obtained from the most preferred criterion restricts the second most preferred criterion and so on. Here, a new objective is added in order to determine the final solution of the NEFs. If the NEFs provided by the BTTA are already unique, the solution will be maintained. Given the optimal solution, $p_{g}^{*}, z^{*}, w^{*}$ of the BTTA, the tariffs' variance is minimized. The choice of this objective function takes into account the coherency of the locational signal. Specifically, in this case, the location signal arises because the final result is only limited by the network constraints, since the power generation dispatch and the minimum tariff amplitude are the same from the previous BTTA optimization problem. Although some alternatives have been investigated (variance maximization and minimization/maximization of the average), the minimization of the variance presented the best results. Also, it follows the same idea as the range minimization: approximate the tariffs to the pro-rata allocation, constrained by the system's locational signals. The formulation of this additional problem is presented in (31)-(39).

$$
\begin{aligned}
& \min _{\substack{\alpha_{g}^{G E N} \\
\alpha_{d g}^{D E M}}} \frac{1}{N^{G E N}+N^{D E M}-1}\left[\sum_{g \in \Omega_{g}}\left(\pi_{g}-m\right)^{2}+\sum_{d \in \Omega_{d}}\left(\pi_{d}-m\right)^{2}\right] \\
& \pi_{g, \pi_{d}} \\
& \begin{array}{c}
m \\
\text { subject to: }
\end{array} \\
& \pi_{g}=\sum_{\ell \in \Omega_{\ell}} \sum_{d \in \Omega_{d}} \frac{C_{\ell}}{2} d_{\ell} \gamma_{g d \ell} \alpha_{g d}^{G E N} ; \quad \forall g \in \Omega_{g} \\
& \pi_{d}=\sum_{\ell \in \Omega_{L}} \sum_{g \in \Omega_{g}} \frac{C_{\ell}}{2} d_{\ell} \gamma_{g d \ell} \alpha_{d g}^{D E M} ; \quad \forall d \in \Omega_{d} \\
& m=\frac{\sum_{g \in \Omega_{g}} \pi_{g}+\sum_{d \in \Omega_{d}} \pi_{d}}{N^{G E N}+N^{D E M}} \\
& \sum_{d \in \Omega_{d}} \alpha_{g d}^{G E N}=1 ; \quad \forall g \in \Omega_{g}
\end{aligned}
$$


$\sum_{g \in \Omega_{g}} \alpha_{d g}^{D E M}=1 ; \quad \forall d \in \Omega_{d}$

$p_{g}^{*} \alpha_{g d}^{G E N}=P_{d} \alpha_{d g}^{D E M} ; \quad \forall g \in \Omega_{g}, \forall d \in \Omega_{d}$

$w^{*} \leq \pi_{g} \leq z^{*} ; \quad \forall g \in \Omega_{g}$

$w^{*} \leq \pi_{d} \leq z^{*} ; \quad \forall d \in \Omega_{d}$

Given this additional step, the flowchart of the proposed TTA process is presented in Fig. 2.

Initializations: network parameters, peak demand, $Z^{M A X}$ and $W^{M I N}$

\begin{tabular}{|c|}
\hline \multicolumn{2}{|c|}{ Bilevel Transmission Tariff Allocation } \\
\hline \multicolumn{1}{|c|}{$p_{g}^{*}, z^{*}, w^{*}$} \\
\hline Minimization of tariffs' variance (31)-(39) \\
$\qquad \alpha_{g d}^{G E N^{*}}, \alpha_{d g}^{D E M^{*}}, \pi_{g}^{*}, \pi_{d}^{*}$ \\
\hline Postage stamps calculation (10)-(11) \\
$\qquad \Delta^{G E N}, \Delta^{D E M}$ \\
\hline Final Tariffs (12)-(13)
\end{tabular}

Fig. 2. Transmission tariff allocation process.

\section{RESULTS}

In this section the performance of the proposed methodology is assessed by using two test systems. A 6-bus system is used to illustrate the NEFs and the power flow decomposition results. Additionally, the IEEE 118-bus system is used to validate the methodology for larger systems. The generation dispatches and tariffs results are discussed.

As mentioned in Section IV, the proposed transmission tariff allocation framework involves nonlinear and quadratic optimization models. In both cases a large scale nonlinear optimization solver CONOPT [22] is used in GAMS [23]. Analysis on both the optimality and the computational performance of CONOPT are also provided. All the simulations are performed on an Intel ${ }^{\circledR}$ Core i7 $3.4 \mathrm{GHz}$ with $8 \mathrm{~GB}$ of RAM.

\section{A. 6-bus System}

The 6-bus system is depicted in Fig. 3. The cost and capacity of the transmission lines $\left(C_{\ell}^{T} ; \operatorname{Cap}_{\ell}\right)$, generation limits $\left(P_{g}^{M A X}\right)$, and the peak demands are presented in Fig. 3. The limit $P_{g}^{M I N}$ is set to 0 . For this system, the TTA process described in Fig. 2 is applied using the arbitrary limits $Z^{M A X}=$ $\$ 7 / \mathrm{MW}$ and $W^{M I N}=\$ 0 / \mathrm{MW}$ as tariff bounds. The running time for the BTTA model applied to this system is approximately $1 \mathrm{~s}$. The tariff range chosen results in a coupled bilevel optimization problem (region II in Fig. 1).

The system's maximum congestion is 723.1 MW that corresponds to $48.2 \%$ of the total system capacity (summation of all lines' capacities). The corresponding optimal generation dispatch is $p_{g}^{*}=[46.3,208.7,385.0]$ MW for generators 1,3 , and 5, respectively. The locational tariffs are $\pi_{g}^{*}=$ $[2.96,4.79,7.00] \$ / M W$ for generators 1,3 , and 5. For demands 2, 4 and 6 the locational tariffs are $\pi_{d}^{*}=$ $[6.20,4.14,7.00] \$ / M W$. Also, the postage stamps calculated by (10)-(11) are $\Delta^{G E N}=\Delta^{D E M}=\$ 12.18 / \mathrm{MW}$. The optimal NEFs are presented in Table I.
Given the power flow decomposition in (7) it is possible to determine the power flow tracing that leads to a certain tariff value. Generator 5 is selected for a detailed analysis since it shows the highest tariff. All the power flow decomposition results that involve generator 5 are presented in Table II.

As it can be seen, the bilateral power exchanges can produce counter-flows that reduce the line congestions, which corresponds to a negative term (reduction) in the tariff. The tariff of generator 5 is the highest due to components $f_{4-5}^{5,4}$ and $f_{5-6}^{5,6}$. Both components are associated to the most expensive transmission lines.

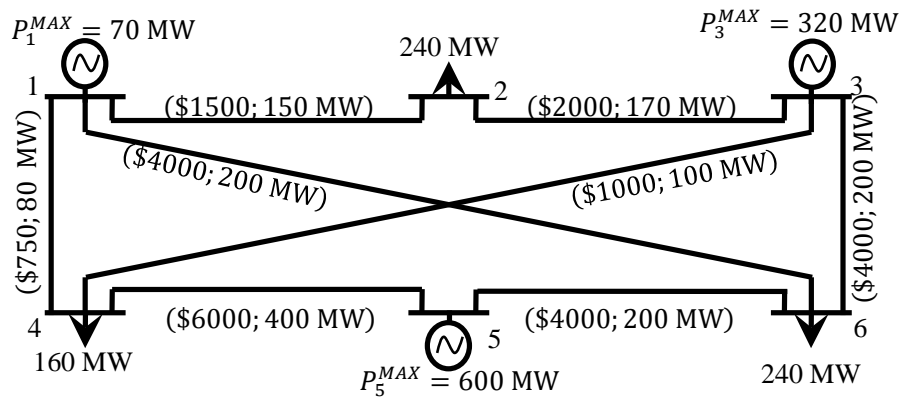

Fig. 3. 6-bus system.

Table I. NEF values for the 6-bus system

\begin{tabular}{|c|c|c|c|}
\hline & Generator 1 & Generator 3 & Generator 5 \\
\hline \multirow{2}{*}{ Demand 2 } & $\alpha_{12}^{G E N}=1.000$ & $\alpha_{32}^{G E N}=0.686$ & $\alpha_{52}^{G E N}=0.131$ \\
& $\alpha_{21}^{D E M}=0.193$ & $\alpha_{23}^{D E M}=0.596$ & $\alpha_{25}^{D E M}=0.211$ \\
\hline \multirow{2}{*}{ Demand 4 } & $\alpha_{14}^{G E N}=0.000$ & $\alpha_{34}^{G E N}=0.000$ & $\alpha_{54}^{G E N}=0.416$ \\
& $\alpha_{41}^{D E M}=0.000$ & $\alpha_{43}^{D E M}=0.000$ & $\alpha_{45}^{D E M}=1.000$ \\
\hline \multirow{2}{*}{ Demand 6 } & $\alpha_{16}^{G E N}=0.000$ & $\alpha_{36}^{G E N}=0.314$ & $\alpha_{56}^{G E N}=0.453$ \\
& $\alpha_{61}^{D E M}=0.000$ & $\alpha_{63}^{D E M}=0.273$ & $\alpha_{65}^{D E M}=0.727$ \\
\hline
\end{tabular}

Table II. Power flow tracing for generator 5 in MW

\begin{tabular}{|c|c|c|c|}
\hline & pair $(5,2)$ & pair $(5,4)$ & pair $(5,6)$ \\
\hline line 1-2 & $f_{1-2}^{5,2}=17.2$ & $f_{1-2}^{5,4}=-0.4$ & $f_{1-2}^{5,6}=0.91$ \\
\hline line 1-4 & $f_{1-4}^{5,2}=8.0$ & $f_{1-4}^{5,4}=-8.8$ & $f_{1-4}^{5,6}=19.10$ \\
\hline line 1-6 & $f_{1-6}^{5,2}=9.2$ & $f_{1-6}^{5,4}=8.4$ & $f_{1-6}^{5,6}=-18.2$ \\
\hline line 2-3 & $f_{2-3}^{5,2}=33.2$ & $f_{2-3}^{5,4}=0.4$ & $f_{2-3}^{5,6}=-0.9$ \\
\hline line 3-4 & $f_{3-4}^{5,2}=12.5$ & $f_{3-4}^{5,4}=-13.8$ & $f_{3-4}^{5,6}=30.0$ \\
\hline line 3-6 & $f_{3-6}^{5,2}=-20.7$ & $f_{3-6}^{5,4}=-14.2$ & $f_{3-6}^{5,6}=30.9$ \\
\hline line 4-5 & $f_{4-5}^{5,2}=20.52$ & $f_{4-5}^{5,4}=137.6$ & $f_{4-5}^{5,6}=49.1$ \\
\hline line 5-6 & $f_{5-6}^{5,2}=29.91$ & $f_{5-6}^{5,4}=22.6$ & $f_{5-6}^{5,6}=125.3$ \\
\hline
\end{tabular}

\section{B. IEEE 118-bus System}

The IEEE 118-bus system is presented in Fig. 4. The complete data information used in the simulations can be found in [24]. Note that generators and demands at the same buses are replaced by the corresponding net power injection and, consequently, the tariffs are nodal. Following the methodology described in Fig. 2, the maximum congestion, the generation dispatch and the final transmission tariffs are obtained and analyzed next. For this system, the average running time for the BTTA model is 4 min for the coupled bilevel model and $16 \mathrm{~s}$ for the decoupled bilevel model.

The optimal tariffs are compared with the ones resulting from the Nodal method. These results are obtained for two different levels of system demand. In the first one, the original system demand is used. In the second, the demand is increased by $20 \%$ in areas I, II and III (shown in Fig. 4) to simulate a more highly congested system. The effects of the demand growth are highlighted. 


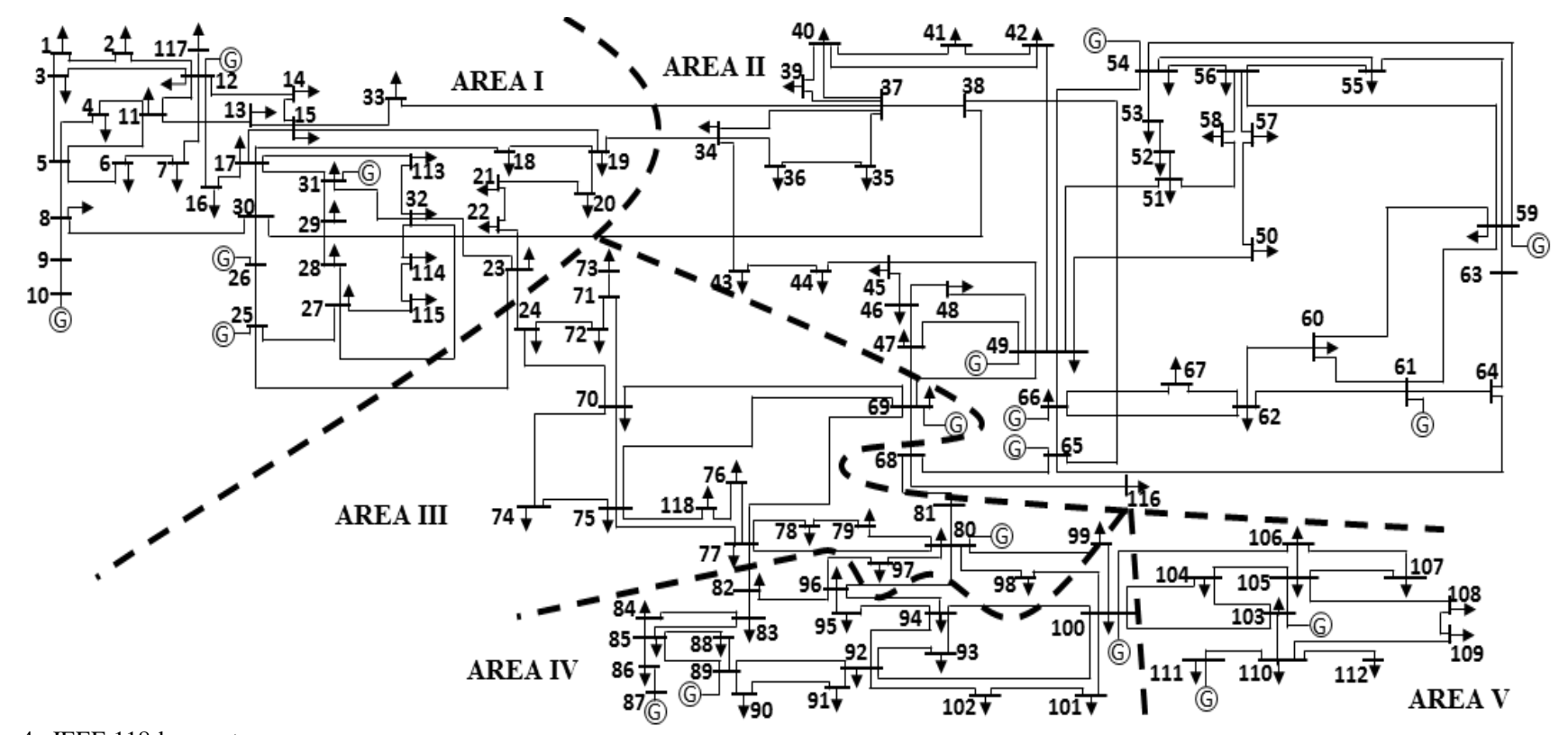

Fig. 4. IEEE 118-bus system.

As discussed in Section IV.B, the optimal system congestion is related to the $Z^{M A X}$ and $W^{M I N}$ bounds. The analysis illustrated in Fig. 1 is reproduced for this test system. Having $W^{M I N}=0$ fixed, the maximum congestion level is obtained for different $Z^{M A X}$ values. The results for the two demand conditions are presented in Fig. 5. The percentages of the total system capacity (summation of all lines' capacities) used by the optimal $y_{W P F}$ are shown.

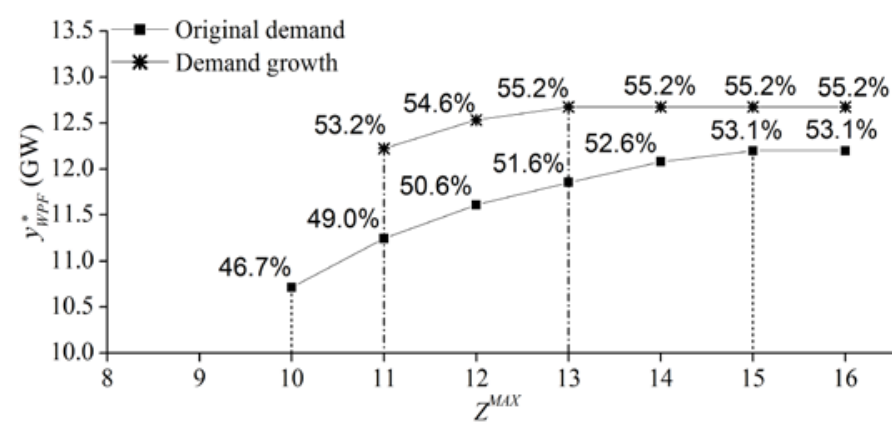

Fig. 5. Maximum congestion of the system as a function of tariff limit $Z^{M A X}$ (with $W^{M I N}=0$ ) in the IEEE 118-bus system.

In Fig. 5, the lowest feasible value of $Z^{M A X}$ for both demand conditions shows the effect of the power flow constraints in the tariffs. From the tariff point of view, a bound value lower than \$10/MW for the original demand and lower than $\$ 11 / \mathrm{MW}$ for the larger demand results in an infeasible problem (region I in Fig. 1). Note that for a more highly congested system (demand increase), the first feasible value of $Z^{M A X}$ is higher and the region for which the BTTA problem is coupled is smaller, as presented in Fig. 5. In an extreme situation of a highly congested system, the BTTA problem can be decoupled irrespectively of the tariff bounds. As each one of the points presented in Fig. 5 corresponds to a tariff allocation, the next results come from the fixed bounds $Z^{M A X}=\$ 12 / \mathrm{MW}$ and $W^{M I N}=\$ 0 / \mathrm{MW}$. The generation dispatches that produce an optimal system use $\left(y_{W P F}\right)$ of $50.6 \%$ and $54.6 \%$ for the original and increased demand conditions are presented in Fig. 6. Also, the generation limits $P_{g}^{M A X}$ are given. The limit $P_{g}^{M I N}$ is set to $10 \%$ of $P_{g}^{M A X}$. This choice implies that all generators are dispatched above $0 \mathrm{MW}$ in the worst-case power flow solution in order to be allocated a nonzero locational tariff to each one of them. However, the proposed model is valid for any other value of $P_{g}^{M I N}$ based on either operation points or historical data.

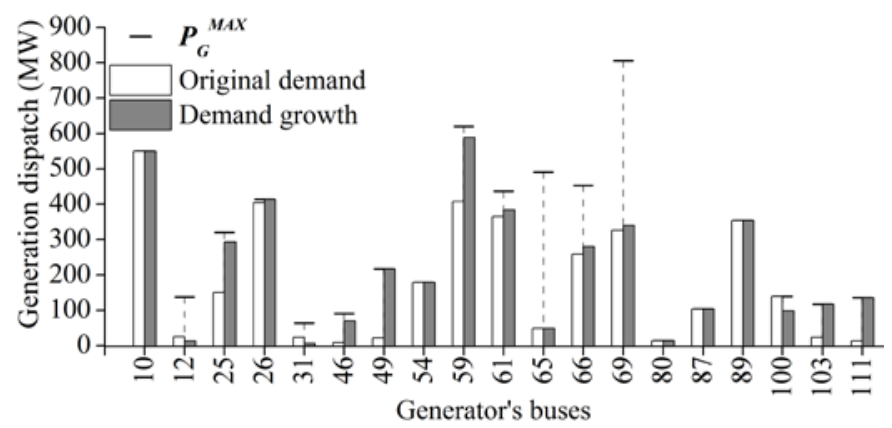

Fig. 6. Generation dispatches for the original and congested IEEE 118-bus system, with $Z^{M A X}=\$ 12 / \mathrm{MW}$ and $W^{M I N}=\$ 0 / \mathrm{MW}$.

Firstly, it can be seen that the optimal maximum congestion dispatch does not follow the $P_{g}^{M A X}$ proportionality rule generally applied [3]. As an example, generator 65 dispatches less power than other generators with lower capacity for the two demand conditions. Also, the changes in the results when the demand grows confirm that the constraints of the proposed model allow for adaptations to modifications. Still in Fig. 6, generators 10 and 59 are isolated from the demand concentration and together supply approximately $28 \%$ of the peak demand in both simulations. This situation simulates the need to apply the TTA criterion adopted in this paper.

The final tariffs results correspond to the sum of the optimized locational tariffs and the postage stamp terms, $\Delta^{G E N}=\Delta^{D E M}=\$ 24.24 / \mathrm{MW}$ for the original system and $\Delta^{G E N}=\Delta^{D E M}=\$ 17.54 / \mathrm{MW}$ for the system with demand growth. A lower postage stamp for the second demand condition is a consequence of the increase in the maximum 
congestion. Postage stamp tariffs are responsible for covering the costs of the lines' overcapacities, which is not used in the power flow solution. When a higher demand takes place, it decreases the transmission lines overcapacity and, consequently, the postage stamp tariff is reduced.

The final tariffs are compared to the Nodal method tariffs, which are obtained from the same peak demand and generation dispatches (Fig. 6). The tariffs for the generators are presented in Fig. 7.

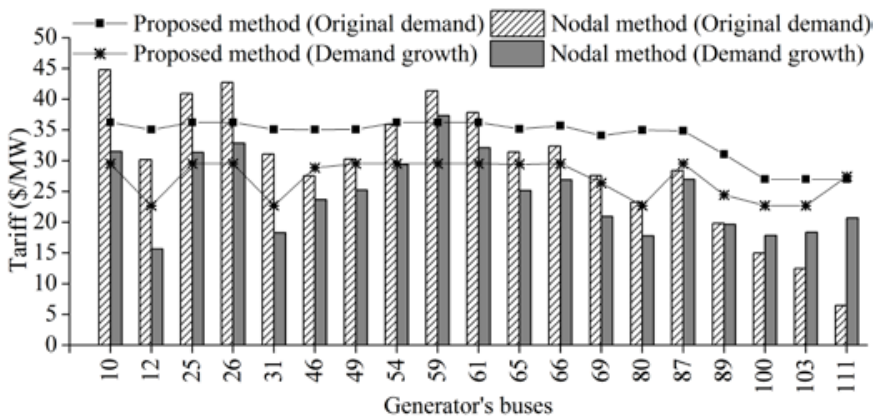

Fig. 7. Generators' final tariffs for the original and demand growth IEEE 118bus system, with $Z^{M A X}=\$ 12 / \mathrm{MW}$ and $W^{M I N}=\$ 0 / \mathrm{MW}$.

In Fig. 7 it can be seen that the proposed method produces tariff groups or levels with the same values. The more congested the system and the stronger the locational signals, the more the proposed method moves away from a unique tariff level (pro-rata allocation). In relation to the range of the tariffs, there is a reduction when the proposed method is compared to the Nodal method. As a consequence, higher tariffs are minimized. In the original demand, generator 10 has the higher tariff for the Nodal method and for the proposed method. However, in the proposed method, the tariff is lower compared to the one obtained by the Nodal method. Analyzing the locational signal coherence, other generators that receive a high tariff in the Nodal method (25, 26 and 59) also receive high tariffs in the proposed method. On the other hand, generators 100, 103 and 111 receive the lowest tariffs in both methods.

When demand grows, overcapacity decreases, the lines are more efficiently used and, as a consequence, the proposed method provides lower tariffs. This is an advantage because, as it can be seen, the more capacity used, the lower the tariffs. For this case, the highest tariff is now allocated to generator 59 in the Nodal and proposed methods, but, in the proposed method, the tariff is lower compared to the Nodal method. With a more highly congested system, stronger locational signals take place and a greater similarity between both methods is observed. More specifically, this occurs for generators $12,31,69,80$ and 111 . Although the results of the Nodal method are not a goal to be reached, a similar locational signal behavior reinforces the idea that the proposed formulation guarantees a coherent locational signal, and at the same time allows minimal tariff range within the bounds $Z^{M A X}$ and $W^{M I N}$.

For the sake of space, the demand tariffs analysis just shows the statistics presented in Table III. The complete results can be seen in [24]. The areas shown in Fig. 4 are used to facilitate the proposed study. The minimum, maximum, average and the variance of the tariffs are presented. The demand growth condition is used and the results are presented in Table III.

Firstly, for all areas, the tariff range in the proposed method is smaller compared to the Nodal method. However, the tariff averages are similar for both methods. In particular, in area 2 a higher variance occurs for both methods, but it is much lower in the proposed method than in the Nodal method. In area 4, a unique tariff level is obtained by the proposed method, which is coherent with the lowest variance of the Nodal method.

Table III. Demand's tariffs statistics in \$/MW - IEEE 118-bus system with demand growth.

\begin{tabular}{|c|c|c|c|c|c|}
\hline \multicolumn{7}{|c|}{ Proposed method } \\
\hline & Area 1 & Area 2 & Area 3 & Area 4 & Area 5 \\
\hline Minimum & 28.12 & 22.71 & 27.15 & 29.53 & 28.06 \\
\hline Maximum & 29.53 & 29.53 & 29.53 & 29.53 & 29.53 \\
\hline Average & 29.31 & 26.97 & 29.24 & 29.53 & 29.02 \\
\hline Variance & 0.25 & 8.19 & 0.49 & 0.00 & 0.52 \\
\hline \multicolumn{7}{|c|}{ Nodal method } \\
\hline \multicolumn{7}{|c|}{ Area 2 } & Area 3 & Area 4 & Area 5 \\
\hline Minimum & 22.48 & 13.70 & 23.14 & 24.30 & 28.10 \\
\hline Maximum & 33.53 & 39.36 & 34.42 & 31.01 & 36.08 \\
\hline Average & 29.24 & 25.83 & 29.79 & 28.73 & 31.47 \\
\hline Variance & 8.43 & 65.83 & 7.03 & 3.22 & 6.21 \\
\hline
\end{tabular}

\section{Computational Performance}

As previously mentioned, the BTTA is a nonconvex nonlinear model with products of variables in the objective function and constraints, which complicates its solution. In this case, an alternative method to achieve the final solution is to use a nonlinear local solver, such as CONOPT [22]. This solver is based on the Generalized Reduced Gradient (GRG) algorithm, which considers an iteration process that computes the reduced gradient of a group of nonbasic variables (selected using the Jacobian of the constraint matrix), which must be below an optimality tolerance for the final optimal solution. For all the simulations, the tolerance used is set to $10^{-7}$.

The main issue in the use of nonlinear local solvers is to guarantee that the global optimal is achieved among the local optima that could have been provided. In this sense, this section compares the CONOPT's BTTA solution with the global optimal solution offered by a linearization technique applicable to small instances of the proposed problem. This linearization is done by the binary expansion technique [25], which approximates continuous decision values by a set of discrete values, formulated as a function of binary variables. Applying this conversion to the decision variables present in the nonlinearities, the nonlinear model is transformed into a linear mixed-integer model. In the case of the BTTA, this needs to be applied only to the $p_{g}$ variable, as it is present in all the nonlinearities. The more precise the approximation and the larger is the problem instance is, the more necessary binary variables are. Considering that the computational effort for solving linear mixed-integer problems grows exponentially with the number of binary variables, in the case of the BTTA, binary expansion is only applicable to small instances. Given this limitation, this section's results consider the 6-bus system presented in Section V.A and its variations.

To solve the nonlinear model via CONOPT, an initial point 
is needed. A good initial point (in the neighborhood of the global optimal) contributes to achieve the desired solution. In the case of the BTTA, the model itself provides a good initialization by first solving the linear problem (1)-(5) to obtain the $p_{g}$ values, and then solving problem (14)-(23) which is also linear since variables $p_{g}$ are fixed to their previous solution. If the chosen limits $Z^{M A X}$ and $W^{M I N}$ are not feasible in the solution of (14)-(23), they can be relaxed to provide the initial point. This initial point is used in the 6-bus system and the results compared to the binary expansion linearization. The precision in the approximation of variable $p_{g}$ is $0.5 \mathrm{MW}, \forall g \in \Omega_{g}$, and the optimality gap in the final integer solution is $0.95 \%$. The results for the main variables are presented in Table IV.

The results confirm that the CONOPT's solution is close to the global optimal obtained by the linearized BTTA. The largest difference, $0.35 \mathrm{MW}$ for variable $y_{W P F}$, is reasonable. To evaluate the computational benefits, the 6-bus system is extended, and the 12, 18, 24 and 30-bus systems are additionally solved. The complete data of each system can be seen in [24]. Table V shows the computing times for each system.

In order to reduce the number of binary variables in the linear mixed-integer BTTA model and hence the running times, a discretization of $10 \mathrm{MW}$ is used to approximate $p_{g}$, $\forall g \in \Omega_{g}$. The results indicate that increasing the number of binary variables in the linear mixed-integer BTTA model causes an exponential increase in the running time compared to the nonlinear BTTA model.

Table IV. Nonlinear and linear mixed-integer solutions for the BTTA model.

\begin{tabular}{|c|c|c|}
\hline & Nonlinear BTTA model & $\begin{array}{c}\text { Linear mixed-integer BTTA } \\
\text { model }\end{array}$ \\
\hline$y_{W P F}$ & 723.06 & 722.71 \\
\hline$z, w$ & $7.000,2.958$ & $6.999,2.961$ \\
\hline$p_{1}, p_{3}, p_{5}$ & $46.330,208.669,385.002$ & $46.375,208.625,384.844$ \\
\hline$\alpha_{12}^{G E N}, \alpha_{14}^{G E N}, \alpha_{16}^{G E N}$ & $1.00,0,0$ & $1.00,0,0$ \\
\hline$\alpha_{32}^{G E N}, \alpha_{34}^{G E N}, \alpha_{36}^{G E N}$ & $0.686,0,0.314$ & $0.686,0,0.314$ \\
\hline$\alpha_{52}^{G E N}, \alpha_{54}^{G E N}, \alpha_{56}^{G E N}$ & $0.131,0.416,0.453$ & $0.131,0.415,0.454$ \\
\hline$\alpha_{21}^{D E M}, \alpha_{23}^{D E M}, \alpha_{25}^{D E M}$ & $0.193,0.596,0.211$ & $0.193,0.597,0.210$ \\
\hline$\alpha_{41}^{D E M}, \alpha_{43}^{D E M}, \alpha_{45}^{D E M}$ & $0,0,1.00$ & $0,0,1.00$ \\
\hline$\alpha_{61}^{D E M}, \alpha_{63}^{D E M}, \alpha_{65}^{D E M}$ & $0,0.273,0.727$ & $0,0.273,0.727$ \\
\hline
\end{tabular}

Table V. Nonlinear and linear mixed-integer computational times for the BTTA model.

\begin{tabular}{|c|c|c|c|c|c|}
\hline \multirow{2}{*}{$\begin{array}{c}\mathrm{n}^{0} \text { of } \\
\text { buses }\end{array}$} & \begin{tabular}{c} 
Nonlinear BTTA model \\
\cline { 2 - 6 } \\
time (s)
\end{tabular} & $\begin{array}{c}\mathrm{n}^{\circ} \text { of } \\
\text { constraints }\end{array}$ & $\begin{array}{c}\text { Linear mixed-integer BTTA model } \\
\text { time }\end{array}$ & $\begin{array}{c}\mathrm{n}^{\circ} \text { of } \\
\text { constraints }\end{array}$ & $\begin{array}{c}\mathrm{n}^{\circ} \text { of binary } \\
\text { variables }\end{array}$ \\
\hline 12 & 2 & 221 & $2 \mathrm{~min}$ & 4573 & 34 \\
\hline 18 & 5 & 416 & $1 \mathrm{~h} 2 \mathrm{~min}$ & 10004 & 51 \\
\hline 24 & 9 & 671 & $40 \mathrm{~h}$ & 17535 & 68 \\
\hline 30 & 15 & 974 & $93 \mathrm{~h}$ & 27154 & 85 \\
\hline
\end{tabular}

\section{CONCLUSIONS}

This paper presents an optimization framework to allocate transmission tariffs among network users under different allocation criteria. In this paper, a criterion has been established to mitigate the impact of the transmission tariffs for generators far away from load centers, which simulates a common problem of isolated renewable generators that occurs in many power systems in the world. This criterion has assumed the WPF condition for which the tariffs' variation range has been minimized.

The BTTA proposed is a bilevel programming problem that maximizes the line congestion in the upper level, taking into account the tariffs characterized by the lower level. The proposed flow-based formulation guarantees that consistent locational tariffs are obtained in the optimization process. The results presented show that the proposed framework is flexible enough to determine both the system congestion level and the locational tariffs under different demand conditions.

\section{REFERENCES}

[1] M. Ilic, F. Galiana, L. Fink, “System Planning Under Competition”, in Power Systems Restructuring: Engineering and Economics. New York, USA: Springer, 1998, pp. 283-334.

[2] D. A. Lima, A. Padilha-Feltrin, J. Contreras, "An overview on network cost allocation methods”, Electr. Power Syst. Res., vol. 79, pp. 750-758, Dec. 2008.

[3] A. Bakirtzis et al., "Comparison of two methods for long-run marginal cost-based transmission use-of-system pricing”, IEE Proc. Gener. Transm. Distrib., vol. 148, no. 4, pp. 477-481, Jul. 2001.

[4] A. J. Conejo, J. Contreras, D. A. Lima, A. Padilha-Feltrin, "Zbus transmission network cost allocation,” IEEE Trans. Power Syst., vol. 22, no. 1, pp. 342-349, Feb. 2007.

[5] J. Bialek, "Topological generation and load distribution factors for supplement charge allocation in transmission open access", IEEE Trans. Power Syst., vol. 12, no. 3, pp. 1185-1193, Aug. 1997.

[6] F. J. Rubio-Odériz, I. J. Pérez-Arriaga, "Marginal pricing of transmission services: a comparative analysis of network cost allocation methods”, IEEE Trans. Power Syst., vol. 15, no. 15, Feb. 2000.

[7] F. F. Wu, Y. Ni, P. Wei, "Power transfer allocation for open access using graph theory-Fundamentals and applications in systems without loop flow”, IEEE Trans. Power Syst., vol. 15, no. 3, Aug. 2000.

[8] G. Strbac, D. Kirschen, S. Ahmed, "Allocating transmission system usage on the basis of traceable contributions of generators and loads to flows”, IEEE Trans. Power Syst., vol. 13, no. 2, May 1998.

[9] F. D. Galiana, A. J. Conejo, H. A. Gil, "Transmission network cost allocation based on equivalent bilateral exchanges", IEEE Trans. Power Syst., vol. 18, no. 4, pp. 1425-1431, Nov. 2003.

[10] Superintendence of Regulation of Transmission Services. (Nov. 1999). Manual da metodologia nodal para cálculo de tarifas de uso dos sistemas elétricos. ANEEL. Brasil. [Online]. Available: http://www.aneel.gov.br/arquivos/PDF/Metodologia_completa.pdf.

[11] D. Shirmohammadi et al., "Evaluation of transmission network capacity use for wheeling transactions", IEEE Trans. Power Syst., vol. 4, no. 4, pp. 1405-1412, Oct. 1989.

[12] Y. Tsukamoto, I. Iyoda, "Allocation of fixed transmission cost to wheeling transactions by cooperative game theory”, IEEE Trans. Power Syst., vol. 11, no. 2, May 1996.

[13] A. R. Abhyankar, S. A. Soman, S. A. Khaparde, "Optimization approach to real power tracing: an application to transmission fixed cost allocation”, IEEE Trans. Power Syst., vol. 21, no. 3, pp. 1350-1361, Aug. 2006.

[14] R. Abhyankar, S. A. Soman, and S. A. Khaparde, "Min-max fairness criteria for transmission fixed cost allocation”, IEEE Trans. Power Syst., vol. 22, no. 4, pp. 2094-2104, Nov. 2007.

[15] M. S. S. Rao, S. A. Soman, P. Chitkara, R. K. Gajbhiye, N. Hemachandra, B. L. Menezes, "Min-max fair power flow tracing for transmission system usage cost allocation: a large system perspective", IEEE Trans. Power Syst., vol. 25, no. 3, pp. 1457-1468, Aug. 2010.

[16] M. S. S. Rao, S. A. Soman, "Marginal pricing of transmission services using min-max fairness policy”, IEEE Trans. Power Syst., vol. 30, no. 2, pp. 573-584, Mar. 2015.

[17] E. Telles, D. A. Lima, A. Street, J. Contreras, "Min-max long run marginal cost to allocate transmission tariffs for transmission users", Electr. Power Syst. Res., vol. 101, pp. 25-35, Aug. 2013.

[18] M. Junqueira, et al., "An Aumann-Shapley approach to allocate transmission service cost among network users in electricity markets", IEEE Trans. Power Syst., vol. 22, no. 4, Nov. 2007. 
[19] E. Telles, D. A. Lima. "Transmission Tariff Allocation under High Renewable Source Penetration and Congestion Conditions”, in Proc. of Power Systems Computation Conference (PSCC). Wrocław, Poland, Aug. 18-22, 2914, pp. 1-8.

[20] PJM. (2010, Mar.). A Survey of Transmission Cost Allocation Issues, Methods and Practices. PJM. United States. [Online]. Available: http://ftp.pjm.com/ /media/documents/reports/20100310-transmissionallocation-cost-web.ashx

[21] A. V. Zykina, "A lexicographic optimization algorithm", Automation and Remote Control, vol. 65, no. 3, pp. 363-368, 2004.

[22] GAMS Development Corporation. GAMS Documentation Center. United States. [Online]. Available: http://www.gams.com/help/index.jsp?topic=\%2Fgams.doc\%2Fsolvers\% 2Findex.html.

[23] GAMS software. [Online]. Available: https://www.gams.com.

[24] E. Telles, "Data of Electric System for Transmission Tariff Simulations”, Maxwell Research Data Repository. [Online]. Available: http://www.maxwell.vrac.pucrio.br/Busca_etds.php?strSecao=resultado\&nrSeq=25585@2.

[25] M. V. Pereira et al., "Strategic bidding under uncertainty: a binary expansion approach”, IEEE Trans. Power Syst., vol. 20, no. 1, pp. 180188, Feb. 2005.

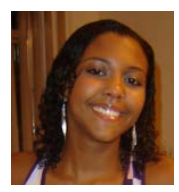

Érica Telles received the B.S. degree, the M.S. degree and Ph.D degree in electrical engineering from Pontifical Catholic University of Rio de Janeiro (PUC-Rio), Rio de Janeiro, Brazil, in 2010, 2012 and 2016. Her research interests include power systems operations and economics, as well as optimization.

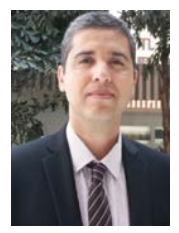

Delberis A. Lima (M'15) holds a M.Sc. and a D.Sc. in Electrical Engineering from the Universidade Estadual Paulista (UNESP), Ilha Solteira, São Paulo, Brazil. Currently, he is associate professor at Pontifical Catholic University of Rio de Janeiro (PUC-Rio). His research interests include power systems planning, operations and economics, smartgrid and electricity markets.

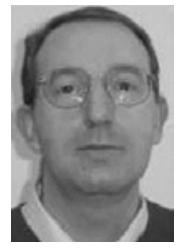

Javier Contreras (SM'05, F'15) received the B.S. degree in electrical engineering from the University of Zaragoza, Zaragoza, Spain, in 1989, the M.Sc. degree from the University of Southern California, Los Angeles, in 1992, and the Ph.D. degree from the University of California, Berkeley, in 1997. He is currently Full Professor at the University of Castilla - La Mancha, Ciudad Real, Spain. His research interests include power systems planning, operations and economics, and electricity markets.

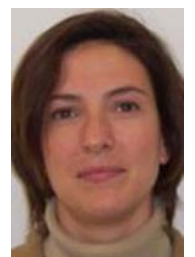

Natalia Alguacil (S’97-M'01-SM’07) received the Ingeniero en Informática degree from the Universidad de Málaga, Málaga, Spain, in 1995, and the Ph.D. degree in power systems operations and planning from the Universidad de Castilla - La Mancha, Ciudad Real, Spain, in 2001. She is currently an Associate Professor of electrical engineering at the Universidad de Castilla - La Mancha. Her research interests include operations, planning, and economics of power systems, as well as optimization. 